\title{
Evaluating the price of tiny kinetic mixing
}

\author{
Tony Gherghetta, ${ }^{1, *}$ Jörn Kersten $\odot,{ }^{2, \dagger}$ Keith Olive, ${ }^{1,3, *}$ and Maxim Pospelov ${ }^{1,3,4,8}$ \\ ${ }^{1}$ School of Physics and Astronomy, University of Minnesota, Minneapolis, Minnesota 55455, USA \\ ${ }^{2}$ University of Bergen, Institute for Physics and Technology, Postboks 7803, 5020 Bergen, Norway \\ ${ }^{3}$ William I. Fine Theoretical Physics Institute, School of Physics and Astronomy, University of Minnesota, \\ Minneapolis, Minnesota 55455, USA \\ ${ }^{4}$ Perimeter Institute for Theoretical Physics, Waterloo, Ontario N2J 2W9, Canada
}

(Received 12 September 2019; published 1 November 2019)

\begin{abstract}
We consider both "bottom-up" and "top-down" approaches to the origin of gauge kinetic mixing. We focus on the possibilities for obtaining kinetic mixings $\epsilon$ which are consistent with experimental constraints and are much smaller than the naive estimates $\left(\epsilon \sim 10^{-2}-10^{-1}\right)$ at the one-loop level. In the bottom-up approach, we consider the possible suppression from multiloop processes. Indeed we argue that kinetic mixing through gravity alone, requires at least six loops and could be as large as $\sim 10^{-13}$. In the top-down approach we consider embedding the Standard Model and a $U(1)_{X}$ in a single grand-unified gauge group as well as the mixing between Abelian and non-Abelian gauge sectors.
\end{abstract}

DOI: 10.1103/PhysRevD.100.095001

\section{INTRODUCTION}

While we can be quite certain of the existence of dark matter (DM), we can with equal certainty claim that we have no idea as to the nature or identity of the dark matter, as it pertains to its connection to fundamental particle physics. This is not because of the lack of options, but rather due to a great multitude of possibilities for DM. Some well-motivated weak-scale candidates such as a fourth-generation heavy neutral lepton [1], have long been excluded by the width of the $Z$ gauge boson [2] and direct detection experiments [3-5]. However, most DM models have been only partially constrained, rather than outright excluded. This includes supersymmetric DM candidates [6,7] that so far have been absent in LHC searches [8-11], and in direct detection experiments [12-14]. Ultralight DM, including axions [15-17], could be another generic option, but no positive evidence for DM of this kind has emerged thus far either.

Given the lack of a clear top-down preference for DM, an alternative approach has been pursued in recent years, that consists of investigating simple UV-complete theories of particle DM. This approach has led to the concept of "dark sectors," which include not only the DM particles but also

\footnotetext{
tgher@umn.edu

joern.kersten@uib.no

*olive@umn.edu

\$mpospelov@perimeterinstitute.ca
}

Published by the American Physical Society under the terms of the Creative Commons Attribution 4.0 International license. Further distribution of this work must maintain attribution to the author(s) and the published article's title, journal citation, and DOI. Funded by SCOAP. possible force carriers that allow the DM to interact with itself and/or with the Standard Model (SM) [18-20]. Constrained only by the fundamental principles of gauge invariance, anomaly cancellation etc., such an approach leaves many possibilities open, and usually does not predict the strength of the interaction from first principles. This can be contrasted with the framework provided by supersymmetry, where the interaction strength can often be fixed from first principles. Indeed, one of the attributes of supersymmetry as an extension of the SM is the specific nature of the interactions between the new particles and SM particles, as they are all related to gauge or Yukawa interactions using known supersymmetric transformations. Although very difficult to detect, even the gravitino interactions with matter can be predicted.

In the dark sector approach, the interaction of DM with the SM can occur through one (or several) portals. For the classification and current experimental constraints, see e.g., the recent reviews [21,22]. The phenomenology of new Abelian gauge bosons, as possible mediators of DM-SM interactions, has been extensively studied in the literature [23-25]. Being electrically neutral, such new gauge bosons may exist in a wide mass range, from the sub-eV energy scale to the weak scale and beyond. The gauge boson mass may be due to some spontaneous breaking of a dark gauge group, or in the Abelian case may be given by a Stückelberg term in the Lagrangian.

The most natural way of coupling the SM fields to the dark sector is via the so-called kinetic mixing operator. Kinetic mixing occurs whenever a term such as

$$
\mathcal{L} \supset \epsilon \frac{1}{2} F^{\mu \nu} X_{\mu \nu}
$$




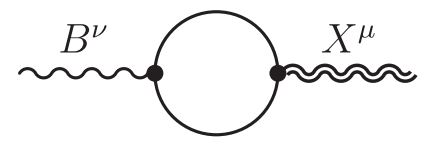

FIG. 1. A Feynman diagram depicting the generation of kinetic mixing at the 1-loop level.

appears in the Lagrangian where $\epsilon$ is a dimensionless parameter. Here $F_{\mu \nu}=\partial_{\mu} A_{\nu}-\partial_{\nu} A_{\mu}$ is the electromagnetic field strength which is related to the $U(1)_{Y}$ hypercharge field strength $B^{\mu \nu}$ via $\cos \theta_{W}$ where $\theta_{W}$ is the weak mixing angle, and $X_{\mu \nu}=\partial_{\mu} X_{\nu}-\partial_{\nu} X_{\mu}$ is the field strength for a hidden sector $U(1)_{X}$ gauge boson, $X_{\mu}$. Assuming that the kinetic mixing vanishes at a high scale and there are fields charged under both $U(1)$ 's, the Feynman diagram in Fig. 1 yields the well-known result $[26,27]$

$$
\epsilon=-\frac{g^{\prime} g_{X}}{16 \pi^{2}} \sum_{i} Y_{i} q_{i} \ln \frac{M_{i}^{2}}{\mu^{2}}
$$

for kinetic mixing with $U(1)_{Y}$ at the one-loop level. Here, $g^{\prime}$ and $g_{X}$ are the gauge couplings of the two $U(1)$ 's, $Y_{i}$ and $q_{i}$ are the respective charges of the fields in the loop with mass $M_{i}$, and $\mu$ is a renormalization scale. In the absence of precise cancellations, this leads to an estimate of $\epsilon \sim\left(10^{-2}-10^{-1}\right) \times g_{X}$, depending on the exact field content of particles running in the loop, and the scale separation in the logarithm. The kinetic mixing with the photon is obtained by multiplying $\epsilon$ by $\cos \theta_{W}$, which does not change the order of magnitude estimate for the mixing. Consequently, to obtain the small amount of mixing required by experimental limits [18-20], we need either a very small gauge coupling for the new $U(1)_{X}$ or an alternative mechanism which generates kinetic mixing.

In fig. 2, we show the strongest bounds on $\epsilon$ as a function of the dark photon mass. These limits come from a variety of sources which include the magnetic field of Jupiter [28], the cosmic microwave background [29,30], searches for deviations from Coulomb's law [31], the CERN Resonant WISP Search (CROWS) [32,33], extra energy loss of stars [34-36], effects of dark photon decay on cosmology [37], SN1987A [38], as well as fixed target experiments and searches for dilepton resonances [22].

We see that the limits on the kinetic mixing parameter at the sub-GeV scale are below the value found at one loop, which is thus too large for many phenomenological applications. Notable examples of constraints on $\epsilon$ include the above mentioned astrophysical constraints on a eV-to$100 \mathrm{keV}$ mass $X$ boson, where the constraint on $\epsilon$ can be as tight as $10^{-15}[34,35]$. In addition, DM masses in the range of 10 to $100 \mathrm{MeV}$ and $X$-mediated freeze-out often require values for the kinetic mixing between $10^{-5}$ and $10^{-3}$ $[41,42]$, which are also in tension with the one-loop estimate. Also note that Fig. 2 refers to the limits on $\epsilon$

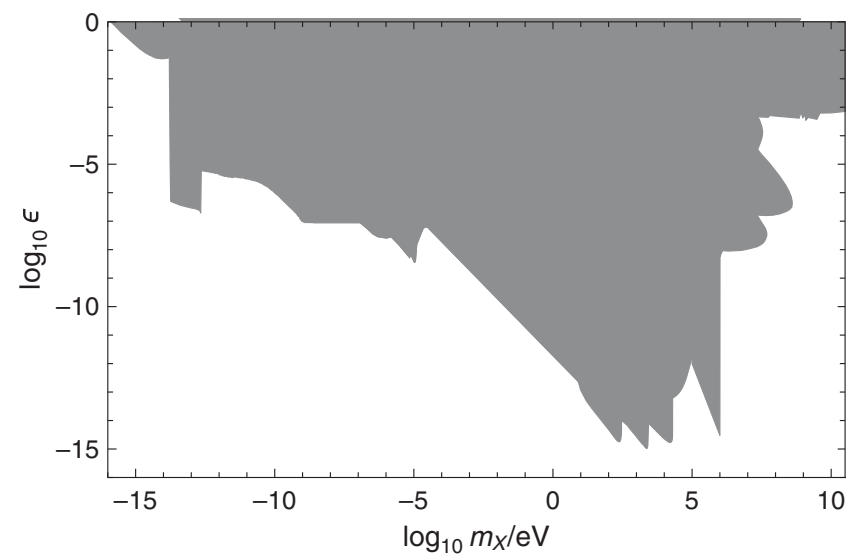

FIG. 2. A summary of the experimental bounds on kinetic mixing, showing the strongest available bound for each dark photon mass $m_{X}$. Adopted from [39]. Not shown are the additional "islands" of CMB- and BBN-excluded regions extending down to $\epsilon \sim 10^{-18}$ for $m_{X}$ in the MeV-range [40].

when the $X$ gauge boson has a Stückelberg-type mass. A dark Higgs origin for $m_{X}$ results in a stronger bound in the entire range $m_{X} \lesssim 10 \mathrm{keV}$, where the combination $\epsilon \times g_{X}$ is limited to $\lesssim 10^{-14}$ from the energy loss by dark Higgs emission in stars, in particular red giants [43].

While a phenomenological (or "bottom-up") approach does not single out any particular value for $g_{X}$ and $\epsilon$, significant restrictions on their value may come from a theoretical requirement of gauge coupling unification. While there are different ways of embedding the SM in a grand unified theory (GUT), there are few attempts for augmenting the SM with a new "dark" $U(1)$ gauge group. One of the questions we wish to address in this paper is the level of kinetic mixing any new gauge interaction may have with the SM (the photon in particular), in the context of a GUT.

If the SM is unified into a GUT, the hidden gauge bosons may be embedded at some scale into a GUT gauge group larger than $S U(5)$. If not, kinetic mixing with the unified field strength will require the presence of effective operators coupling the adjoint representation of the GUT with the hidden sector. We will discuss both of these possibilities with a view of estimating how large or small kinetic mixing may be.

The GUT-based approach, interpreted naively as $\alpha_{X} \sim \alpha_{\mathrm{SM}}$, may not be inevitable in the top-down approach. Indeed, in the literature, LARGE volume string compactifications have been pointed out as a way to obtain very small gauge couplings $g \sim 10^{-4}$ (or $\alpha \sim 10^{-9}$ ) [44] and tiny kinetic mixing via Eq. (2) [45]. Alternatively, in string theory extra $U(1)$ 's are ubiquitous either from the closed string sector [46] (including e.g., RR photons [47]) or open string hidden sectors [48], and these can mix with the visible sector.

Independent of any GUT, we explore the phenomenological ranges of kinetic mixing that may receive additional suppression from multiloop mechanisms. Surprisingly, 
kinetic mixing may also occur through purely gravitational interactions, provided that there is a source of charge symmetry breaking in the dark sector. We argue that this particular type of mixing through gravity requires at least six loops. Although heavily suppressed by the gravitational coupling and loop factors, a non-negligible mixing of order $10^{-13}$ is possible with a Planck scale cutoff. Furthermore we argue that this is the minimum kinetic mixing in any theory with hidden gauge interactions and charge symmetry breaking in the dark sector.

The outline of this paper is as follows: We begin with a survey of phenomenological (bottom-up) approaches to kinetic mixing, including possible multiloop generation mechanisms. In particular we discuss mechanisms via graviton exchange, and point out the conditions needed to generate this particular type of kinetic mixing. In Sec. III A, we survey the various top-down possibilities for grand unification which includes the hidden sector. The generation of effective operators that mix an extra $U(1)_{X}$ with a SM GUT is discussed in Sec. III B. Our conclusions are given in Sec. IV.

\section{PHENOMENOLOGICAL (BOTTOM-UP) APPROACHES}

In this section, we consider some ideas for generating kinetic mixing using a bottom-up approach, demonstrating a wide variety of possibilities. However before we do that, some general comments based on symmetry arguments are in order. Consider the schematic Lagrangian

$$
\mathcal{L}=\mathcal{L}_{A}+\mathcal{L}_{X}+\mathcal{L}_{\text {int }}(X, A),
$$

that includes two "separate" Lagrangians, $\mathcal{L}_{A, X}$ which contain kinetic terms for gauge bosons and their interaction with currents built from matter fields, $\mathcal{L}_{A}=-\frac{1}{4} F_{\mu \nu}^{2}-$ $A^{\mu} J_{\mu}^{(A)}+\cdots$ Here $J_{\mu}^{(A)}$ is the current of particles charged only under a $U(1)_{A}$ gauge group. The interaction Lagrangian between the two sectors can include kinetic mixing as well as other generic forms of interactions between the fields charged under $U(1)_{A}$ and $U(1)_{X}$. One can introduce two separate charge conjugation symmetries, $\mathcal{C}_{A}$ and $\mathcal{C}_{X}$ that act on the fields as $\mathcal{C}_{A}(A)=-A$, $\mathcal{C}_{X}(X)=-X$. The operator $F^{\mu \nu} X_{\mu \nu}$ is obviously odd under these separate charge symmetry transformations. Notice that if $X$ is massless and there is no matter charged under $X$, the kinetic mixing operator can be removed by a $(A, X)$ field redefinition. In this case, even in the presence of the kinetic mixing operator, one can define two independently conserved charge conjugation symmetries. However the introduction of a mass term, $m_{X}^{2} X_{\mu}^{2}$, makes $\epsilon$ observable, so that it is the $\epsilon \times m_{X}^{2}$ parameter that breaks two charge symmetries down to one common $\mathcal{C}$.

If $\mathcal{C}_{A}$ and $\mathcal{C}_{X}$ are separately good symmetries of the full Lagrangian, then kinetic mixing cannot be induced at any perturbative order [46,49]. In order to generate kinetic mixing, the individual charge symmetries must be broken, either completely or down to a common charge symmetry. For example, if both $\mathcal{L}_{A}$ and $\mathcal{L}_{X}$ are QED-like, then $\mathcal{C}_{A}\left(\mathcal{L}_{A}\right)=\mathcal{L}_{A}$ and $\mathcal{C}_{X}\left(\mathcal{L}_{X}\right)=\mathcal{L}_{X}$. If in addition the interaction term $\mathcal{L}_{\text {int }}$ is also invariant under separate charge symmetries, then the kinetic mixing term cannot be generated.

As an explicit example, consider two scalar QED theories with one field $\phi$ charged under $A$, and another field $\chi$ charged under $X$ with an interaction Lagrangian in the form of a scalar portal, $\mathcal{L}_{\text {int }}=-\lambda\left(\phi^{\dagger} \phi\right)\left(\chi^{\dagger} \chi\right)$. In such a theory, the full Lagrangian $\mathcal{L}$ is invariant under separate charge conjugation symmetries, and therefore kinetic mixing will never develop at any perturbative order because at least one of the $\mathcal{C}$ symmetries would need to be violated, either in $\mathcal{L}_{A, X}$ or in $\mathcal{L}_{\text {int }}$.

The one-loop example from the previous section demonstrates that commonly charged matter does indeed break individual charge conjugation symmetries down to a common $\mathcal{C}$-symmetry. In other words, matter interactions with both gauge bosons, e.g., $\bar{\psi} \gamma_{\mu} D_{A X}^{\mu} \psi$, where $D_{A X}^{\mu}$ is the covariant derivative with respect to the $A$ and $X$ fields, cannot be made separately $\mathcal{C}_{A}$ and $\mathcal{C}_{X}$ symmetric. This interaction is of course invariant under a usual charge conjugation symmetry: $\mathcal{C}\left(\bar{\psi} \gamma_{\mu} \psi\right)=-\bar{\psi} \gamma \gamma_{\mu} \psi$, under which both fields are transformed, $\mathcal{C}(X)=-X$, and $\mathcal{C}(A)=-A$.

Moreover, the charge conjugation symmetry is indeed maximally violated in the SM, as is parity, due to a drastic asymmetry in the charge assignments between the left- and right-handed fields. However this does not mean that kinetic mixing will be induced for any "dark" gauge boson $X$, as $\mathcal{C}_{X}$ must also be broken. Therefore the most crucial assumptions affecting the kinetic mixing depend on the structure of the dark $X$-sector (QED-like or chiral, SM-like) and the presence or absence of commonly charged matter fields. In all the examples considered below, we will assume that the separate $\mathcal{C}_{X}$ symmetry is violated.

\section{A. Gauge-mediated kinetic mixing}

We begin with the one-loop estimate of Holdom, Eq. (2), and "work our way down" in $\epsilon$ by pursuing different choices of $X$ interactions. What are the generic ways of making the kinetic mixing $\epsilon$ smaller without assuming the gauge couplings are tiny?

In the bottom-up picture, we do not have any information about the tree-level value of $\epsilon$ at very high energies, which is determined by unknown UV physics. We are therefore restricted to determining the radiative corrections in the low-energy theory. These can be viewed either as the result of the running of $\epsilon$ from high to low energy or as loop corrections evaluated directly at the low-energy scale relevant for observations. The results will usually depend on an unphysical renormalization scale $\mu$, as in Eq. (2), for example. As long as we do not specify the precise observable sensitive to $\epsilon$, it is not obvious which value 
to choose for $\mu$. However, since $\mu$ only appears logarithmically, this does not introduce an uncertainty of more than an order of magnitude, which is sufficient for our purposes.

We will consider the value of the lowest-order nonzero correction to $\epsilon$ as a generic lower limit. Of course, smaller values can be obtained if there is a cancellation between a nonzero tree-level value and radiative corrections. ${ }^{1}$

One obvious possibility for suppressing $\epsilon$ is to introduce several particles in the commonly charged sector in such a way that the sum in Eq. (2) is small. If, for example, there are two heavy matter fields, $\psi$ and $\chi$, with the same charges under one gauge group and opposite charges under the other, then the kinetic mixing parameter is suppressed. Indeed, at a loop momentum scale much above the particle masses, the sum gives zero, and only threshold effects due to $m_{\psi \cdot \chi}$ give a nonzero result. Thus, in this case we will have $\sum_{i=\psi, \chi} Y_{i} q_{i} \ln \left(M_{i}^{2} / \mu^{2}\right)$ simplifying to $Y_{\psi} q_{\psi} \ln \left(M_{\psi}^{2} / M_{\chi}^{2}\right)$ (or more precisely to a difference of polarization diagrams for $\chi$ and $\psi$ ). In the limit of degenerate masses, the logarithm can be very small, approximately $\Delta M^{2} / M^{2}$, where $M$ is the common mass scale and $\Delta M$ is the mass splitting. Such a mass degeneracy could result from an underlying GUT symmetry, as further discussed in Sec. III A. Similar effects are also found in string theory, and result from an underlying mass degeneracy in the string spectrum [46].

With the exception of matter fields with degenerate masses, kinetic mixing generated at one-loop is generically too large for the phenomenological applications discussed in the introduction. This suggests trying to realize the suppression of $\epsilon$ by devising a multi-loop generation mechanism. A known example is the mirror-symmetric twin Higgs model, where kinetic mixing is at least fourloop-suppressed, leading to $\epsilon \sim 10^{-13}-10^{-10}$ [51,52].

We begin with two loops, and it turns out that it is not entirely trivial to find a working example. Consider the generic two-loop diagram in Fig. 3. If we choose $U(1)_{Y} \times U(1)_{X}$ charges $(q, 0)$ for $\psi$ and $(0, q)$ for $\chi$, we obtain kinetic mixing if $\phi$ has charges $(q,-q)$, while the one-loop diagram of Fig. 1 with $\psi$ or $\chi$ in the loop cannot contribute. However, the analogous one-loop diagram with $\phi$ in the loop does contribute and will lead us back to the estimate (2).

A working example can be obtained at the three-loop level by using the neutrino portal between active (SM) and sterile (SM-singlet) neutrinos. We consider a $U(1)_{X}$ gauge boson that couples only to the sterile neutrino sector. In addition to the "standard" Yukawa interaction $y_{N} L H N_{i}$ (with Yukawa coupling $y_{N}$ ) that couples heavy singlet neutrinos $N_{i}$, with Majorana mass $m_{N}$, to the SM Higgs $H$ and lepton doublet $L$, we introduce the $y_{X} N_{i} H_{X} N_{X}$ portal (with Yukawa coupling $y_{X}$ ) that further couples $N_{i}$ to a Higgs field $H_{X}$ and a fermion $N_{X}$ charged under $U(1)_{X}$ [53]. The typical

\footnotetext{
${ }^{1}$ Such fine-tuning can have the upside of an interesting cosmology [50].
}

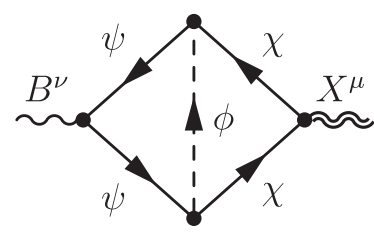

FIG. 3. A Feynman diagram depicting the generation of kinetic mixing at the 2-loop level.

mass hierarchy is $m_{N} \gg m_{W} \gg m_{X}>m_{N_{X}}{ }^{2}$ Kinetic mixing will be induced as shown on the left in Fig. 4, and we estimate

$$
\epsilon \sim \frac{y_{N}^{2} y_{X}^{2} g_{X} g^{\prime}}{\left(16 \pi^{2}\right)^{3}} \ln \frac{\mu^{2}}{m_{N}^{2}} \sim 10^{-7}\left(y_{N} y_{X}\right)^{2},
$$

assuming the log factor is of order one.

By cutting the internal $H_{X}$ line, we can form a dimensionsix operator, $B^{\mu \nu} X_{\mu \nu} H_{X}^{\dagger} H_{X}$ and after replacing both $H_{X}$ 's with the dark Higgs vacuum expectation value (vev), $v_{X}$, we obtain a two-loop diagram shown on the right in Fig. 4, which gives a contribution of similar size, depending on parameter values. It is important to note that the result is now proportional to the Yukawa couplings $y_{N}$ and $y_{X}$. Therefore, the size of the kinetic mixing can be dialed to an almost arbitrarily small value, by choosing $y_{N} y_{X}$ to be very small (although doing so, may cause other model dependent problems with multiple very light fermions).

In the effective theory valid below the electroweak scale, which corresponds to the model considered in [54,55], the three-loop diagram in Fig. 4 can be reduced to the two-loop diagram shown in Fig. 5 with a four-Fermi vertex. After the electroweak symmetry and $U(1)_{X}$ are broken, SM neutrinos mix with $N_{i}$ and $N_{X}$. Although kinetic mixing with the photon cannot be generated at one loop since there is no field with both an electric and a $U(1)_{X}$ charge, it can instead arise from Fig. 5. A very rough estimate is

$$
\epsilon \sim \frac{e g_{X}}{\left(16 \pi^{2}\right)^{2}} G_{F} m_{X}^{2} \theta^{2} \sim 10^{-17}\left(\frac{m_{X}}{1 \mathrm{MeV}}\right)^{2}\left(\frac{\theta}{0.1}\right)^{2}
$$

where $\theta$ is the active-sterile neutrino mixing angle (e.g., $\theta \sim y_{N} v / y_{X} v_{X}$ if the masses of $N_{i}$ are similar and $m_{N_{X}} \gg m_{\nu}$ ) and we have assumed $g_{X} \sim e \sim 1$.

Next we discuss mechanisms that use not only charged matter but also intermediate gauge bosons of an additional third group. Consider a bottom-up model with two sufficiently heavy vectorlike fermions $\psi$ and $\chi$ as well as an additional gauge group $U(1)_{M}$ that is spontaneously broken at a high scale. The charge assignments are specified in Table I.

\footnotetext{
${ }^{2}$ Note that at least two $N_{i}$ are needed to avoid a massless state.
} 

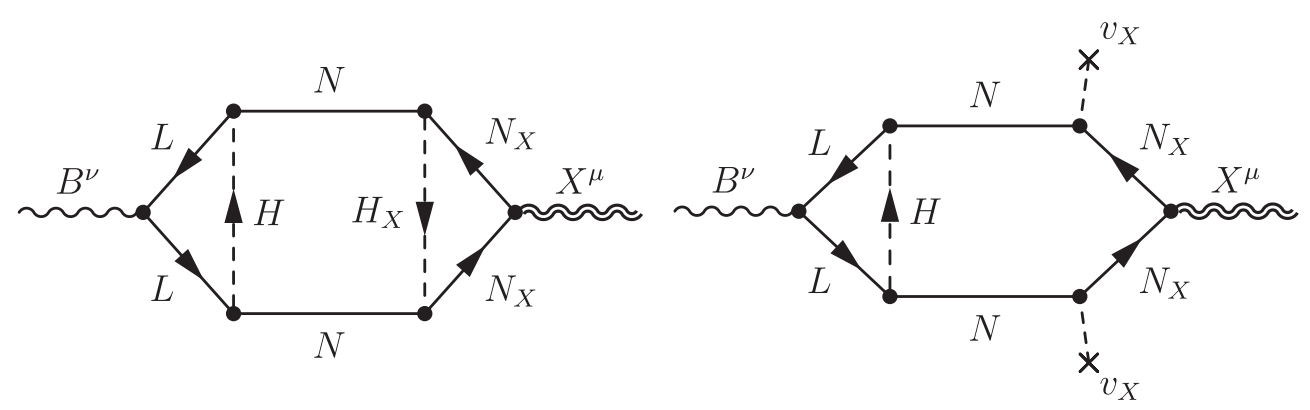

FIG. 4. The Feynman diagrams depicting the generation of kinetic mixing in a neutrino portal model.

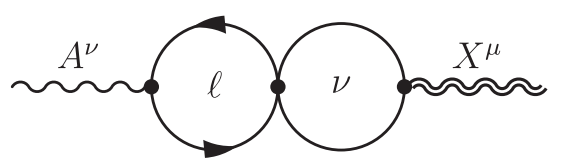

FIG. 5. The generation of kinetic mixing in the low-energy effective theory arising from the neutrino portal model. The particles in the loops are a charged lepton $\ell$ and a neutrino mass eigenstate $\nu$, which is a mixture of a SM neutrino, $N_{X}$, and $N$.

The two-loop diagram in Fig. 6 is proportional to $\Pi_{\mathrm{YM}}^{\nu \rho}\left(k^{2}\right) D_{M}^{\rho \sigma} \Pi_{M X}^{\sigma \mu}\left(k^{2}\right) \sim k^{4} / m_{M}^{2}$, where $\Pi$ denotes a selfenergy contribution. Consequently, this diagram leads to an operator containing derivatives of $B^{\mu \nu}$ and $X^{\mu \nu}$ and thus does not contribute to kinetic mixing. The corresponding three-loop contribution with a second $U(1)_{M}$ gauge boson vanishes due to Furry's theorem (diagrams containing a closed fermion loop with an odd number of vertices do not contribute). Consequently, the leading contribution to kinetic mixing stems from the four-loop diagram in Fig. 6, which is of course highly suppressed,

$$
\epsilon \sim \frac{g^{\prime} g_{X} g_{M}^{6}}{\left(16 \pi^{2}\right)^{4}} \sim 10^{-9}
$$

where $g_{M}$ is the $U(1)_{M}$ gauge coupling. A similar mechanism for generating kinetic mixing was discussed recently in [56], with the intermediate gauge group corresponding to a Yang-Mills field.

TABLE I. The particle content of a bottom-up model yielding kinetic mixing at the 4-loop level. Here "light" refers to mass scales at the electroweak scale and below, while "heavy" refers to mass scales significantly above the weak scale.

\begin{tabular}{lcccc}
\hline \hline & & \multicolumn{3}{c}{ Charge } \\
\cline { 3 - 5 } & Mass & $U(1)_{Y}$ & $U(1)_{M}$ & $U(1)_{X}$ \\
\hline$\psi$ & Heavy & 1 & 1 & 0 \\
$\chi$ & Heavy & 0 & 1 & 1 \\
$B^{\mu}$ & Light & 0 & 0 & 0 \\
$M^{\mu}$ & Heavy & 0 & 0 & 0 \\
$X^{\mu}$ & Light & 0 & 0 & 0 \\
\hline \hline
\end{tabular}

\section{B. Gravity-mediated kinetic mixing}

So far we have considered the outcome for the kinetic mixing parameter $\epsilon$, when there exist matter fields commonly charged under both the SM and the dark $U(1)_{X}$ or another new gauge group. We have seen that there is considerable freedom in the choice of the mediation mechanism, and as a consequence, in the expected value of $\epsilon$.

In this subsection, we would like to address the question of how gravitational interactions alone could result in a finite kinetic mixing parameter. We imagine a series of diagrams that join the SM and the $U(1)_{X}$ sector by gravitational interactions, i.e., loops of gravitons. The size of such diagrams is controlled by some $n$th power of the gravitational constant, $G_{N} \equiv M_{\mathrm{Pl}}^{-2}$. The dimensionless nature of $\epsilon$ tells us that such diagrams may indeed be UV divergent, and one could expect the result to scale as $\propto \Lambda_{\mathrm{UV}}^{2 n} / M_{\mathrm{Pl}}^{2 n}$. Since the UV cutoff, $\Lambda_{\mathrm{UV}}$ could be comparable to the Planck mass $M_{\mathrm{Pl}}$, the extreme smallness of the denominator can be mitigated by a larger numerator, rendering this to be a very UV-sensitive mechanism.

First we consider a case when the SM is supplemented by a noninteracting dark $U(1)_{X}$. While the charge conjugation symmetry is broken in the SM, as discussed earlier, there is a separate charge conjugation symmetry, $\mathcal{C}_{X}$ in the dark sector, $X_{\mu \nu} \rightarrow-X_{\mu \nu}$ that leaves the action invariant (for instance, the dark sector could be QED-like). At the perturbative level this means that any vertex between the gravitons and the $X$-boson will contain an even number of gauge fields, $X_{\mu}$. Therefore, the perturbative result in this case is $\epsilon=0$. Since gravity is expected to preserve both discrete and gauge symmetries, we do not expect this conclusion to change even at a nonperturbative level.

If, on the other hand, there exists some matter content of the dark sector that results in a separate breaking of the dark charge conjugation symmetry, then there is a possibility of inducing nonzero kinetic mixing by means of gravity mediation. Consider, for example, a theory that contains a "mirror" SM-like sector, $\mathrm{SM}^{\prime}$, but no commonly charged fields under any of the SM and $\mathrm{SM}^{\prime}$ gauge groups. Schematically, the action of such a theory can be approximated by the sum of three terms, 


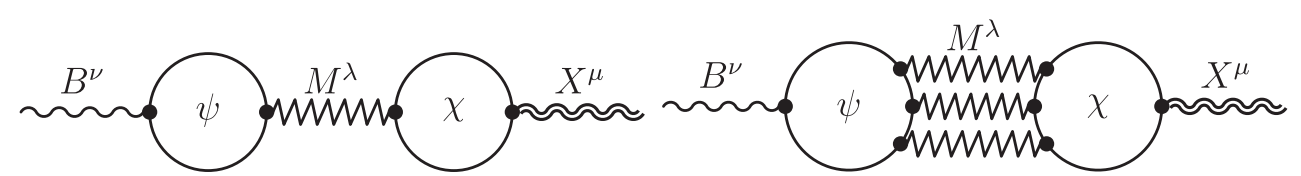

FIG. 6. The generation of kinetic mixing at the 4-loop level. The left Feynman diagram does not yield the correct operator and hence gives no contribution to kinetic mixing.

$$
\mathcal{S}=\mathcal{S}_{\mathrm{SM}}+\mathcal{S}_{\mathrm{SM}^{\prime}}+\mathcal{S}_{\text {gravity }}
$$

Both $\mathrm{SM}$ and $\mathrm{SM}^{\prime}$ necessarily participate in gravitational interactions, such that a diagram schematically shown in Fig. 7 is always possible. The middle section of this diagram connecting two fermion loops in the SM and $\mathrm{SM}^{\prime}$ sectors contains an unknown number of gravitons, $h^{\rho \sigma}$.

It turns out that the minimum number of such intermediate gravitons is three. The best way of showing this is by cutting the diagram through the intermediate gravitons, and representing the left- and right-handed parts as effective operators composed of the $U(1)$ field strength and gravitationally gauge invariant operators. To be gauge invariant, these operators must be composed of the metric $g$ and gauge-invariant derivatives of the metric, i.e., the curvature $\mathcal{R}$ :

$$
F_{\mu \nu} \times \mathcal{O}^{\mu \nu} ; \quad \mathcal{O}=\mathcal{O}(g, \mathcal{R})
$$

It is easy to see that for one or two intermediate graviton exchanges the operator $\mathcal{O}^{\mu \nu}$ either does not exist or can be reduced to a total derivative, such that the operator $F_{\mu \nu} \mathcal{O}^{\mu \nu}$ would not lead to kinetic mixing. For one intermediate graviton all possible candidate structures for $\mathcal{O}^{\mu \nu}$ must contain at most one power of the curvature, such as $g^{\mu \nu}$; $\mathcal{R}^{\mu \nu} ; \nabla^{\mu} \nabla^{\nu} \mathcal{R}$ etc., where $\nabla^{\mu}$ is the gravitational covariant derivative. All of these structures are $\mu \leftrightarrow \nu$ symmetric, and give zero upon contraction with either $X_{\mu \nu}$ or $F_{\mu \nu}$. For two intermediate gravitons, we also find that the required $\mathcal{O}^{\mu \nu}$ tensors do not exist. The following candidate structures are explicitly symmetric under the interchange of indices contracted with the $U(1)$ field strength $F_{\mu \nu}: \mathcal{R}^{\mu \alpha \beta \nu} \mathcal{R}_{\alpha \beta}$, $\mathcal{R}^{\mu \alpha} \mathcal{R}_{\alpha}^{\nu}$. Expressions that contain extra derivatives, such as $\mathcal{R}^{\mu \alpha} \nabla_{\alpha} \nabla^{\nu} \mathcal{R}$ and $\mathcal{R}^{\mu \alpha} \nabla^{2} \mathcal{R}_{\alpha}^{\nu}$ can be simplified using integration by parts, and the result is either $\mu \leftrightarrow \nu$ symmetric, or contains $\nabla F$, and therefore does not lead to kinetic mixing.

Finally, at order $\mathcal{R}^{3}$, one can indeed find the required operators $\mathcal{O}^{\mu \nu}$ that do not vanish. These include structures

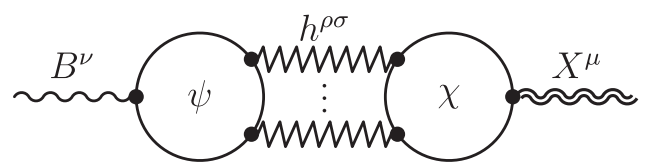

FIG. 7. The mediation of kinetic mixing via gravity, where the dots represent an unknown number of additional gravitons. like $\mathcal{R}_{\alpha}^{\mu} \mathcal{R}_{\lambda \rho} \mathcal{R}^{\nu \lambda \rho \alpha}$ and many other possible terms with derivatives. Such operators would generically lead to three graviton two-loop exchanges generating $\epsilon$. Moreover, the absence of a gravitational anomaly means that the sum of the respective hypercharges of all fermions in the SM and $\mathrm{SM}^{\prime}$ is zero. Therefore to avoid a null result the matter loops contain not only a fermionic loop, but also require an exchange by for example, the Higgs and Higgs' fields inside the fermionic loops, as shown in Fig. 8, so that $\operatorname{Tr}\left(Y_{i} y_{i}^{2}\right) \neq 0$, where $Y_{i}$ are the $U(1)$ charges and $y_{i}$ are the Yukawa couplings.

This raises the loop count to $\mathbf{6}$, and we have the following extremely crude estimate:

$B_{\mu \nu} X_{\alpha \beta}\left\langle\mathcal{O}^{\mu \nu} \mathcal{O}^{\alpha \beta}\right\rangle \rightarrow \epsilon_{\text {grav }} \propto g^{\prime} g_{X} y_{t}^{2} y_{X}^{2}\left(\frac{1}{16 \pi^{2}}\right)^{6} \times \frac{\Lambda_{\mathrm{UV}}^{6}}{M_{\mathrm{Pl}}^{6}}$,

where $g^{\prime}\left(g_{X}\right)$ are the $U(1)\left(U(1)_{X}\right)$ gauge couplings, $y_{t}$ is the top Yukawa coupling, and $y_{X}$ is the Yukawa coupling in $\mathrm{SM}^{\prime}$. In this expression, $\langle\ldots\rangle$ stands for the result of the gravitational loop mediation of the $\mathcal{R}$-containing operators.

If $\Lambda_{\mathrm{UV}}$ is of the same order as the Planck mass, the gravitationally-induced kinetic mixing estimated in (9) could be as large as $\epsilon_{\text {grav }} \sim 10^{-13}$. Interestingly, probing such a small kinetic mixing observationally is not out of the question: astrophysical probes of $\epsilon$ can be very sensitive, particularly if the dark sector mass scale is in the eV-to-keV range [57]. At the same time it is worth mentioning that in theories with a parametrically large number of species, e.g., when the SM is extended by $\mathcal{N}$-copies, one also expects that $\Lambda_{\mathrm{UV}}^{2} \lesssim M_{\mathrm{Pl}}^{2} \times \mathcal{N}^{-1}$, and the proposals of Refs. [58,59] are perhaps not challenged by this mechanism.

\section{Clockwork mechanisms}

The clockwork mechanism was proposed to generate very small couplings in the absence of small fundamental parameters [60]. In its gauge theory implementation, we

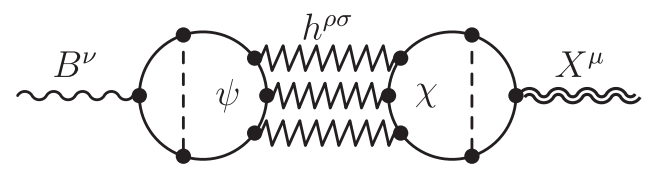

FIG. 8. The mediation of kinetic mixing via gravity showing the minimal three graviton exchange as well as the Higgs exchange inside the fermion loops to prevent a null result from gravitational anomalies. 
consider $N+1 U(1)$ symmetries labeled by $i=0, \ldots, N$ with corresponding gauge fields $A_{\mu}^{i}$ and equal gauge couplings, $g$. The gauge symmetry is broken to a single $U(1)$ by the (equal) vevs $\left\langle\phi_{j}\right\rangle=f / \sqrt{2}$ (for all $j=0, \ldots, N-1)$ of $N$ Higgs fields $\phi_{j}$. Each of these scalars has charges $(1,-q)$ under $U(1)_{j} \times U(1)_{j+1}$ (and charge 0 under the other groups). Diagonalizing the mass matrix for the gauge bosons yields a massless zero mode, the gauge boson of the unbroken $U(1) \equiv U(1)_{X}$. Once this group is broken as well, this field becomes the hidden photon. If a field is charged only under $U(1)_{N}$, its coupling to the hidden photon is exponentially suppressed, $g_{\text {eff }}=\frac{N_{0} g}{q^{N}}$, where $N_{0} \sim 1$ is a normalization factor.

Likewise, if the $U(1)_{Y}$ gauge boson kinetically mixes only with $A_{\mu}^{N}$, its kinetic mixing with the hidden photon is suppressed,

$$
\epsilon_{\text {eff }}=\frac{N_{0} \epsilon}{q^{N}} .
$$

Thus, we can use the gauge clockwork mechanism to generate a tiny kinetic mixing starting from $\epsilon \sim g \sim 1$. The required number of clock gears is given by

$$
N=\left\lceil\log _{q} \frac{N_{0} \epsilon}{\epsilon_{\text {eff }}}\right\rceil,
$$

where $\lceil x\rceil$ denotes the ceiling, i.e., the smallest integer larger than $x$. The result is shown in Fig. 9 as a function of $q$ for $N_{0}=1$ and two different values of $\epsilon_{\text {eff }}$. For example, $\epsilon_{\text {eff }} \sim 10^{-7}$ requires $N=24$ for $q=2$.

As quite a few $U(1)$ 's are needed for a significant suppression, we might consider the continuum limit $N \rightarrow \infty$, in which case the clockwork mechanism becomes equivalent to a 5-dimensional theory with localized bulk gauge bosons [61] and Higgs fields. In this case the suppression factor becomes $e^{-k L}$, where $L$ is the size of

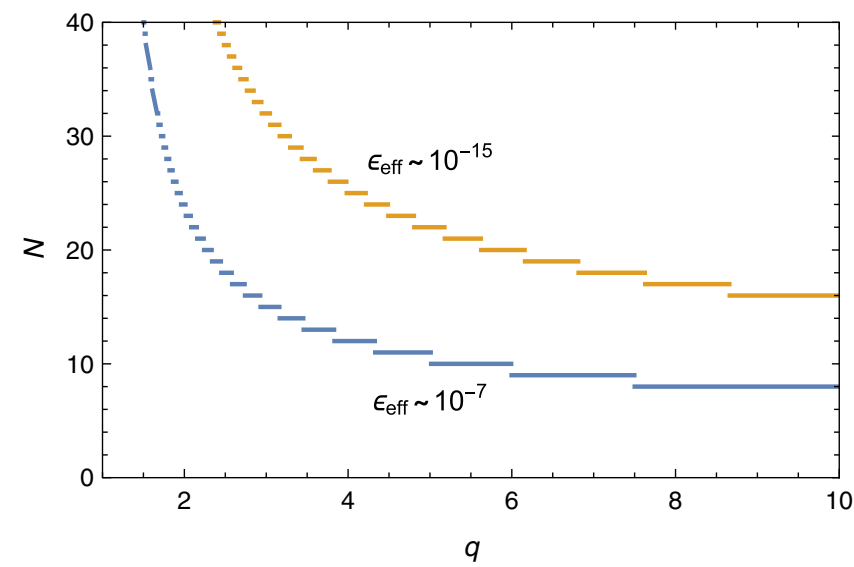

FIG. 9. The number of $U(1)$ gauge groups required to obtain the given kinetic mixing parameter $\epsilon_{\text {eff }}$ via the clockwork mechanism as a function of the charge $q$. the extra dimension (for an orbifold $L=\pi R$ with $R$ the radius of the extra dimension), and $k$ is the equivalent of $q$.

To summarize this section, we remark that the bottom-up approach leaves enough flexibility to cover a wide range of values of the mixing parameter $\epsilon$. Indeed, the one-loop result can be turned into a multiloop generation mechanism. Moreover, in certain examples given in this section, the kinetic mixing parameter vanishes if some corresponding Yukawa couplings vanish. Since Yukawa couplings are not necessarily fixed by unification, one could exploit some features of these mechanisms even within a GUT framework.

\section{THEORETICAL TOP-DOWN APPROACHES}

The bottom-up approaches discussed so far have the disadvantage that they can only provide lower limits on the size of kinetic mixing because they do not contain mechanisms ensuring $\epsilon=0$ at tree level (i.e., forbidding the term $F_{\mu \nu} X^{\mu \nu}$ in the original Lagrangian). In addition, these lower limits can be avoided by a fine-tuned cancellation between a nonzero tree-level value and the loop contributions considered above. We note that when the $U(1)_{X}$ gauge group is embedded in a GUT, we cannot assume a Stiickelberg mass for the dark photon. Instead, we must assume the presence of a dark Higgs of similar mass in which case the stronger limits on $\epsilon$ discussed earlier apply. This will in addition require fine-tuning beyond that already needed for the doublet-triplet splitting in $S U(5)$, in order to obtain a light $H_{X}$. We now turn to top-down models where the absence of kinetic mixing at a highenergy scale is guaranteed by a symmetry.

\section{A. Embedding in a single group}

Let us first assume that both the SM gauge group and $U(1)_{X}$ are embedded in the same group. This implies that the rank of the group is 5 or larger. In this case realistic symmetry breaking patterns often lead to light states that are charged under both $U(1)_{Y}$ and $U(1)_{X}$, and consequently to large kinetic mixing via Fig. 1. However, for sufficiently large groups, it is possible to construct counterexamples. In what follows, we consider progressively large gauge groups and their symmetry breaking patterns and comment on their suitability for generating kinetic mixing. In particular, we try to identify which group and field content could account for mixing below the 1-loop estimate.

$S O(10) \rightarrow S U(5) \times U(1)_{X}:$ The $S O(10)$ multiplet $\mathbf{1 6}$ decomposes into $(\mathbf{1},-5)+(\overline{\mathbf{5}}, 3)+(\mathbf{1 0},-1)$ of $S U(5) \times U(1)_{X}$ [62], where the SM matter fields are contained in the $\overline{\mathbf{5}}$ and 10, which are both charged under $U(1)_{X}$. Equivalently, all SM matter is charged under $U(1)_{B-L}$, which is related to $U(1)_{X}$ via $B-L=\frac{2}{5} Y-\frac{1}{5} X$. Consequently, in this case we 
cannot obtain a kinetic mixing parameter much below the Holdom estimate (2).

$E_{6} \rightarrow S O(10) \times U(1)_{X}$ : The $E_{6}$ multiplet 27 decomposes into $(\mathbf{1},-4)+(\mathbf{1 0}, 2)+(\mathbf{1 6},-1)$, where the SM matter fields are in the $\mathbf{1 6}$ and charged under $U(1)_{X}$. Thus we would again obtain kinetic mixing at the 1-loop level.

$E_{6} \rightarrow S O(10) \times U(1)_{A} \rightarrow S U(5) \times U(1)_{A} \times U(1)_{B}:$ In this case we have two dark $U(1)$ groups at our disposal, which allows us to choose $U(1)_{X}$ as a linear combination of $U(1)_{A}$ and $U(1)_{B}$ such that either the $\overline{\mathbf{5}}$ or the $\mathbf{1 0}$ of $S U(5)$ is uncharged under $U(1)_{X}$. However, as these multiplets stem from the same 16 of $S O(10)$, they have the same $U(1)_{A}$ charge, whereas their $U(1)_{B}$ charges are different (see first item). As a consequence, one multiplet, either the $\overline{\mathbf{5}}$ or the 10, unavoidably ends up with a nonzero charge under both the SM $U(1)$ and $U(1)_{X}$.

$E_{7} \rightarrow E_{6} \times U(1)_{A} \rightarrow S O(10) \times U(1)_{A} \times U(1)_{B}$ : We can again choose $U(1)_{X}$ as a linear combination of $U(1)_{A}$ and $U(1)_{B}$. In this case, we can ensure that the complete 16 of $S O(10)$ inside the 27 of $E_{6}$ is uncharged. Using LIEART [63] we find that the $E_{7}$ multiplet 56 decomposes into $(\mathbf{1}, 3)+(\mathbf{1},-3)+$ $(\mathbf{2 7},-1)+(\overline{\mathbf{2 7}}, 1)$. Hence, the decomposition of the 27 under $S O(10) \times U(1)_{A} \times U(1)_{B}$ is $(\mathbf{1},-1,-4)+$ $(\mathbf{1 0},-1,2)+(\mathbf{1 6},-1,-1)$. Consequently, the choice $X=A-B$ leads to a vanishing $U(1)_{X}$ charge for all light matter fields that arise from the $\mathbf{1 6}$.

However, the light Higgs belongs to a $\mathbf{1 0}$ of $S O(10)$, which is usually assumed to arise from the same $E_{6}$ and $E_{7}$ multiplets as the $\mathbf{1 6}$ containing the matter fields. In this case, Higgs and matter multiplets have the same $U(1)_{A}$ charge but different $U(1)_{B}$ charges, so their $U(1)_{X}$ charges cannot vanish simultaneously and we again return to the Holdom estimate, this time due to a Higgs loop. To avoid this conclusion, we have to embed the $\mathbf{1 0}$ containing the Higgs into a larger multiplet of $E_{6}$ in such a way that the ratio of $U(1)_{A}$ and $U(1)_{B}$ charges for this $\mathbf{1 0}$ is equal to the ratio for the matter 16. Using LIEART we find that this is possible if the $\mathbf{1 0}$ stems from the $\mathbf{1 3 3}$ of $E_{7}$ (which is the smallest representation beyond the 56). This multiplet decomposes into $(\mathbf{1}, 0)+(\mathbf{2 7}, 2)+$ $(\overline{\mathbf{2 7}},-2)+(\mathbf{7 8}, 0)$ of $E_{6} \times U(1)_{A}$, so the decomposition of the $\mathbf{2 7}$ is $(\mathbf{1}, 2,-4)+(\mathbf{1 0}, 2,2)+(\mathbf{1 6}, 2,-1)$. Now $X=A-B$ guarantees that the $U(1)_{X}$ charge vanishes for the $\mathbf{1 0}$ as well.

To summarize this example, we can ensure the vanishing of the 1-loop diagram for kinetic mixing in an $E_{7}$ GUT if we assume that (unlike more typical models of $E_{6}$ unification) the $S O(10)$ Higgs multiplet (a 10) originates from a different $E_{7}$ multiplet than matter. Matter fields sit inside the $\mathbf{1 6}$ of $S O(10)$, which sits inside a 27 of $E_{6}$, which sits inside the $\mathbf{5 6}$ of
$E_{7}$. The $\mathbf{1 0}$ containing the Higgs also resides in a $\mathbf{2 7}$ of $E_{6}$, however, the latter originates from a 133 of $E_{7}$. In this case, there are no light fields with nonzero charges under $U(1)_{X}$.

$E_{8} \rightarrow E_{6} \times S U(3):$ All SM fields can be assigned to the $E_{8}$ multiplet $\mathbf{2 4 8}$, which decomposes into $(\mathbf{1}, \mathbf{8})+(\mathbf{2 7}, \mathbf{3})+(\overline{\mathbf{2 7}}, \overline{\mathbf{3}})+(\mathbf{7 8}, \mathbf{1})$, where $(\mathbf{2 7}, \mathbf{3})$ can accommodate the Higgs and matter fields. If we break $S U(3)$ to the $U(1)_{X}$ that is generated by the diagonal $S U(3)$ generator $\lambda_{3}=\operatorname{diag}(1,-1,0)$, there is an uncharged state in the triplet. If in addition the other two states obtain GUT-scale masses in the course of the symmetry breaking, all light states remain uncharged under $U(1)_{X}$. While this example, is simpler and all SM fields reside in a common $\mathbf{2 7}$ of $E_{6}$, we are forced to a larger unification group and parent representation. In addition, in many $E_{8}$ unification models, the $S U(3)$ subgroup plays the role of a (gauged) family symmetry so that all three matter generations reside in the $(\mathbf{2 7}, \mathbf{3})$. That is not the case here, and we must require a separate $\mathbf{2 4 8}$ for each generation.

$E_{8} \rightarrow S U(5) \times S U(5):$ We assume that the second $S U(5)$ contains $U(1)_{X}$ and we consider the $E_{8}$ representations 248 and $\mathbf{3 8 7 5}$. The options for the SM matter multiplets are $(\overline{\mathbf{5}}, \mathbf{1 0}),(\overline{\mathbf{5}}, \mathbf{1 5}),(\overline{\mathbf{5}}, \mathbf{4 0})$, $(\mathbf{1 0}, \mathbf{5})$, and $(\mathbf{1 0}, \mathbf{4 5})$. Considering the decompositions of 5 and 45 under $S U(5) \rightarrow S U(4) \times U(1)_{X}$ and $S U(5) \rightarrow S U(3) \times S U(2) \times U(1)_{X}$, we find that there are no states uncharged under $U(1)_{X}$. However, if we do not restrict ourselves to maximal subgroups, we can proceed as in the previous item and break $S U(5)$ to one of the $U(1)$ subgroups under which for example, the multiplets $\mathbf{5}$ and $\mathbf{1 0}$ contain uncharged states.

While the next two examples are not specifically unified gauge groups, they have often been considered as UV extensions of the SM.

$S U(4) \times S U(2)_{\mathrm{L}} \times S U(2)_{\mathrm{R}}$ : The usual breaking to the SM by a $(\mathbf{4}, \mathbf{1}, \mathbf{2})$ does not leave an extra $U(1)_{X}$. If we use a 15 instead to break $S U(4) \rightarrow S U(3) \times U(1)_{X}$ (which yields the left-right symmetric model), $X=$ $B-L$ and again all SM matter fields are charged.

$S U(3)_{c} \times S U(3)_{\mathrm{L}} \times S U(3)_{\mathrm{R}}$ : In the minimal trinification model [64], symmetry breaking proceeds via two $(\mathbf{1}, \mathbf{3}, \overline{\mathbf{3}})$ scalars. Individually, each vev breaks the gauge group to $S U(3)_{c} \times S U(2)_{\mathrm{L}} \times S U(2)_{\mathrm{R}} \times U(1)$, but the two scalars lead to different $S U(2)_{\mathrm{R}} \times U(1)$ groups. Consequently, in combination the vevs break $S U(3)^{3}$ directly to the SM gauge group, leaving only a global $U(1)$. Thus, a dark photon and kinetic mixing would require a significantly modified scalar sector that leaves a local $U(1)$ unbroken.

If there are light fields charged under both $U(1)$ 's, they are contained in complete GUT multiplets and then the 
diagram in Fig. 1 vanishes for equal masses. However, this does not decrease $\epsilon$ significantly at low energies, where it will contain logarithms of particle masses, which are not small for the SM particles (cf. Sec. II A).

In any case, heavy fields charged under both $U(1)_{Y}$ and $U(1)_{X}$ will occur. As they fill out complete GUT multiplets, their contribution to $\epsilon$ is sensitive to the mass splittings within these multiplets caused by the GUT symmetry breaking. If this leads to a mass splitting at tree level, we still obtain a sizable value of $\epsilon$ via Eq. (2). However, if the mass degeneracy is only broken by renormalization group running, kinetic mixing arises effectively at the twoloop level, so we expect only $\epsilon \sim 10^{-6}-10^{-4}$ [65]. This is still too large to satisfy some experimental bounds, but an additional suppression by one order of magnitude due to a small coupling could be sufficient when $m_{X} \gtrsim 1 \mathrm{MeV}$.

In summary, among commonly considered unified groups we find examples without light fields charged under $U(1)_{Y, X}$ only for $E_{7}$ and $E_{8}$. We do not attempt to work out the model building details for these cases, which would also have to address the emergence of chiral fields from the real representations of $E_{7}$ and $E_{8}$ (as could, for example, arise from an orbifold compactification).

\section{B. Mixing between non-Abelian and Abelian sectors}

If only one of the gauge groups involved is non-Abelian, the kinetic mixing term $G^{\mu \nu} X_{\mu \nu}$ is forbidden by gauge invariance, since the non-Abelian field strength $G^{\mu \nu}$ is not gauge-invariant. Thus, the diagram of Fig. 1 vanishes even in the presence of particles that are charged under both gauge groups. However, we can realize kinetic mixing via effective operators involving appropriate scalar representations, for example, $\frac{1}{\Lambda} \Sigma G^{\mu \nu} X_{\mu \nu}$, if the scalar $\Sigma$ transforms under the adjoint representation and develops a vev [65]. Such operators have to be generated via loops involving particles of mass $\Lambda$.

The non-Abelian group could be either the dark sector gauge group or a group containing $U(1)_{Y}$. We will focus on the latter option, as it allows for grand unification and implies a simpler dark sector, and will briefly return to the former option afterwards.

\section{Adjoint scalar}

Consider first a dark $U(1)_{X}$ and a visible sector with a GUT gauge group $G \supset U(1)_{Y}$, whose gauge bosons are denoted by $G^{\mu}$. We introduce a scalar $\Sigma$ that transforms under the adjoint representation of the non-Abelian group and is uncharged under $U(1)_{X}$. In addition, we introduce a vectorlike fermion $\psi$ with mass $\Lambda$ that transforms nontrivially under both $G$ and $U(1)_{X}$. Then the diagram in Fig. 10 generates the effective operator $\frac{1}{\Lambda} \Sigma G^{\mu \nu} X_{\mu \nu}$. This diagram can be drawn for any group $G$ and any (nonsinglet) representation of $\psi$, since the coupling of $\psi$ to the adjoint scalar is the same as the coupling to the gauge bosons of $G$ (up to a factor of $\gamma^{\mu}$ ).

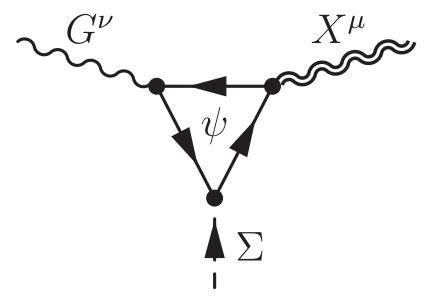

FIG. 10. The generation of an effective operator at the 1-loop level involving an adjoint scalar $\Sigma$ and a vectorlike fermion $\psi$ that leads to kinetic mixing.

Once $\Sigma$ develops a vev $\langle\Sigma\rangle$ (chosen such that the SM gauge group remains unbroken) we obtain kinetic mixing between $B^{\mu}$ and $X^{\mu}$. Assuming that $\langle\Sigma\rangle$ is also responsible for the breaking of the GUT group, the vev $\langle\Sigma\rangle$ is of order the unification scale $M_{\mathrm{GUT}}$, leading to the estimate

$\epsilon \sim \frac{g g_{X} y_{\Sigma}}{16 \pi^{2}} \frac{\langle\Sigma\rangle}{\Lambda} \sim \frac{g g_{X} y_{\Sigma}}{16 \pi^{2}} \frac{M_{\mathrm{GUT}}}{\Lambda} \gtrsim \frac{y_{\Sigma}}{16 \pi^{2}} \frac{M_{\mathrm{GUT}}}{M_{\mathrm{Pl}}} \sim 10^{-4} y_{\Sigma}$,

for $\mathcal{O}(1)$ gauge couplings, where $g$ is the GUT gauge coupling and $y_{\Sigma}$ is the coupling of $\psi$ to $\Sigma$. Thus, to satisfy experimental bounds additional suppression is required and can be obtained most easily by setting the Yukawa coupling $y_{\Sigma}$ to a sufficiently small value.

\section{Fundamental and other representations}

Using a scalar $\phi$ transforming under a representation different from the adjoint, we can generate the effective operator $\frac{1}{\Lambda^{2}} \phi^{\dagger} G^{\mu \nu} \phi X_{\mu \nu}$ via the diagram in Fig. 11. If the unified group is broken by an adjoint vev, the contribution from $\phi$ will be subdominant compared to the one from the adjoint unless $y_{\Sigma} \lesssim y_{\phi}^{2} \frac{\langle\phi\rangle^{2}}{\Lambda\langle\Sigma\rangle}$. Let us explore the possibilities arising in this case. Of course, there are many possible choices, but not every possibility that is allowed by group theory is phenomenologically viable.

For example, consider $G=S U(5)$ with a fundamental scalar $\phi \sim(\mathbf{5}, 0)$, where the numbers in parentheses indicate the $S U(5)$ representation and the $U(1)_{X}$ charge. Then the diagram in Fig. 11 can be realized, for instance, with the vectorlike fermions $\psi \sim\left(\mathbf{5}, q_{\psi}\right)$ and $\chi \sim\left(\mathbf{1 0}, q_{\psi}\right){ }^{3}$ However, as $\phi \sim(\mathbf{3}, \mathbf{1})+(\mathbf{1}, \mathbf{2})$ under $S U(3)_{\mathrm{c}} \times S U(2)_{\mathrm{L}}$, the vev of $\phi$ can only be nonzero for the electrically neutral component, the analog of the SM neutrino in the fermionic $\overline{5}$ multiplet. This component couples to both $B^{\mu \nu}$ and $W_{3}^{\mu \nu}$ in such a way that after electroweak symmetry breaking it has a nonzero coupling only to $Z^{\mu \nu}$ but not to $F^{\mu \nu}$. Consequently, this case is not interesting for us, since it does not lead to kinetic mixing of $X^{\mu}$ with the photon.

\footnotetext{
${ }^{3}$ The diagram can be drawn with different $U(1)_{X}$ charge assignments $q_{\chi} \neq q_{\psi}$ as well, but then $\phi$ needs a nonzero charge, which implies $\langle\phi\rangle \lesssim m_{X}$ and makes it impossible to obtain observable kinetic mixing.
} 


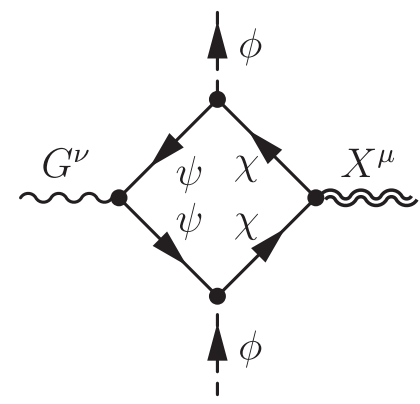

FIG. 11. The generation of an effective operator leading to kinetic mixing, utilizing vectorlike fermions $\psi$ and $\chi$ as well as a scalar $\phi$ transforming under a representation different from the adjoint.

Moreover, $\phi \sim \mathbf{5}$ of $S U(5)$ cannot have a GUT-scale vev since all its components are charged under the SM gauge group. With an electroweak-scale vev and $\Lambda \sim M_{\mathrm{GUT}}$, the contribution to any kinetic mixing is suppressed by $\left(\frac{v_{\mathrm{EW}}}{M_{\mathrm{GUT}}}\right)^{2} \sim 10^{-28}$ and thus much smaller than the minimal contribution from gravity discussed in Sec. II B. Thus, in order to obtain kinetic mixing of a relevant size in cases involving a SM nonsinglet scalar, we would have to lower $\Lambda$ much below $M_{\mathrm{GUT}}$.

As a consequence, we restrict our attention to scalar multiplets that contain a SM singlet and can thus obtain a large vev yielding a sizable $\epsilon$ even if $\Lambda \gtrsim M_{\mathrm{GUT}}$. Sticking to $S U(5)$, the smallest viable multiplet is the $\mathbf{7 5} .{ }^{4}$ Then the smallest fermion multiplet we can use is $\psi=\chi \sim\left(\mathbf{1 0}, q_{\psi}\right)$. Giving a vev (only) to the SM-singlet component of $\phi$, the only nonzero term in the decomposition of $\left\langle\phi^{\dagger}\right\rangle G^{\mu \nu}\langle\phi\rangle$ is the one containing $G_{24}^{\mu \nu}=B^{\mu \nu}$. Hence, we generate kinetic mixing with $B^{\mu}$ (but not $W_{3}^{\mu}$ ) and thus with both the photon and the $Z$, as desired. Its size is of order

$$
\begin{aligned}
\epsilon & \sim \frac{g g_{X} y_{\phi}^{2}}{16 \pi^{2}} \frac{\langle\phi\rangle^{2}}{\Lambda^{2}} \sim \frac{g g_{X} y_{\phi}^{2}}{16 \pi^{2}}\left(\frac{M_{\mathrm{GUT}}}{\Lambda}\right)^{2} \\
& \gtrsim \frac{y_{\phi}^{2}}{16 \pi^{2}}\left(\frac{M_{\mathrm{GUT}}}{M_{\mathrm{Pl}}}\right)^{2} \sim 10^{-6} y_{\phi}^{2},
\end{aligned}
$$

for $\mathcal{O}(1)$ gauge couplings and $\langle\phi\rangle \sim M_{\mathrm{GUT}}$, where now $y_{\phi}$ is the coupling between $\phi, \psi$ and $\chi$. As a result, an additional suppression by one or two orders of magnitude due to small couplings or a smaller value of $\langle\phi\rangle$ is sufficient to satisfy the bounds for $m_{X} \lesssim 10^{-4} \mathrm{eV}$ or $m_{X} \gtrsim 1 \mathrm{MeV}$.

In order to give an example with a different unified group as well, let us take $G=S O(10)$. Then two simple possibilities to realize the diagram of Fig. 11 are $\phi \sim(\mathbf{1 2 6}, 0)$, $\psi \sim\left(\overline{\mathbf{1 6}}, q_{\psi}\right), \chi \sim\left(\mathbf{1 6}, q_{\psi}\right)$, and $\phi \sim(\mathbf{1 6}, 0), \psi \sim\left(\mathbf{1 6}, q_{\psi}\right)$,

\footnotetext{
${ }^{4}$ We note that the $\mathbf{7 5}$ has been used instead of the adjoint $\mathbf{2 4}$ to break $S U(5)$ in the missing partner mechanism to solve the doublet-triplet problem [66].
}

$\chi \sim\left(\mathbf{1 0}, q_{\psi}\right)$. These cases also offer the option of using fermions in the loop that receive masses $\Lambda \sim M_{\mathrm{GUT}}$ via couplings to additional scalars transforming under $\mathbf{4 5}, \mathbf{5 4}$ or 210 and developing GUT-scale vevs to break $S O(10) .{ }^{5}$ In this line of thought, $\phi \sim \mathbf{1 2 6}$ may be especially interesting if it obtains a vev of order $10^{10} \mathrm{GeV}$ or larger that also gives a mass to the right-handed neutrinos in the fermionic $\mathbf{1 6}$. According to Eq. (13), $\langle\phi\rangle \sim 10^{10} \mathrm{GeV}$ and $\Lambda \sim M_{\text {GUT }}$ would result in $\epsilon \sim 10^{-14}$ for $\mathcal{O}(1)$ couplings.

\section{Non-Abelian dark sector}

If the gauge group in the dark sector is non-Abelian, we can obtain kinetic mixing with the SM gauge boson $B^{\mu}$ in the same way as for a non-Abelian visible sector. Now the scalars have to be charged under the dark gauge group. If their vevs $\langle\Sigma\rangle$ and $\langle\phi\rangle$ give a mass to the dark photon, they are of order $m_{X} / g_{X}$, which leads to

$$
\epsilon \sim \frac{g_{X} g^{\prime} y_{\Sigma}}{16 \pi^{2}} \frac{\langle\Sigma\rangle}{\Lambda} \sim \frac{y_{\Sigma}}{16 \pi^{2}} \frac{m_{X}}{\Lambda},
$$

for the adjoint scalar case, and

$$
\epsilon \sim \frac{g_{X} g^{\prime} y_{\phi}^{2}}{16 \pi^{2}} \frac{\langle\phi\rangle^{2}}{\Lambda^{2}} \sim \frac{y_{\phi}^{2}}{16 \pi^{2} g_{X}} \frac{m_{X}^{2}}{\Lambda^{2}}
$$

for the case of a scalar not transforming in the adjoint. Now $\Lambda$ cannot be very large if we are to obtain observable kinetic mixing. However, $\Lambda$ has to be large enough to hide the electrically charged fermions $\psi$ and $\chi$ from detection. For $\Lambda>1 \mathrm{TeV},{ }^{6}$ Eq. (14) yields $m_{X} \gtrsim 10^{14} \epsilon \mathrm{eV}$ in the adjoint case with $y_{\Sigma} \sim 1$. For scalars transforming under different representations and $y_{\phi} \sim 1$, Eq. (15) leads to $m_{X} \gtrsim 10^{13} \sqrt{g_{X} \epsilon} \mathrm{eV}$, which allows us to approach the parameter space interesting for fixed target experiments for $\epsilon \sim 10^{-6}$ and $g_{X} \lesssim 10^{-3}$.

In order to obtain a wider range of viable parameters, we can use a scalar that breaks the non-Abelian dark group to $U(1)_{X}$ at a sufficiently high scale, thus decoupling the vev involved in kinetic mixing from the dark photon mass. The minimal possibility is $S U(2)_{X}$ together with an adjoint scalar. A scenario of this kind leading to $\langle\Sigma\rangle \sim 10^{4} \mathrm{GeV}$ and $\Lambda \sim 10^{16} \mathrm{GeV}$, which corresponds to $\epsilon \sim 10^{-14}$ for $\mathcal{O}(1)$ couplings, was presented in [67].

Finally, we can combine the possibilities discussed in this section by considering non-Abelian groups in both sectors. That is, we assume the overall gauge group $G \times G^{\prime}$, where in the simplest scenario $G \supset U(1)_{Y}$ and $G^{\prime} \supset U(1)_{X}$ are broken by the vevs of the adjoint scalars $\Sigma$ and $\Sigma^{\prime}$,

\footnotetext{
${ }^{5}$ Assuming the vectorlike masses that are independent of GUT breaking are subdominant.

${ }^{6}$ Indirect searches for new physics may well set a significantly stronger limit, depending on details of the dark sector.
} 
respectively. In the presence of a vectorlike fermion of mass $\Lambda$ that is charged under both groups, we obtain [68]

$$
\epsilon \sim \frac{g g_{X} y_{\Sigma} y_{\Sigma^{\prime}}}{16 \pi^{2}} \frac{\langle\Sigma\rangle\left\langle\Sigma^{\prime}\right\rangle}{\Lambda^{2}}
$$

As the unification scales in the two sectors are not related in general, $\left\langle\Sigma^{\prime}\right\rangle$ can be much smaller than $M_{\mathrm{GUT}}$, which yields very small values of $\epsilon$ even if all gauge and Yukawa couplings are of order 1. For example, $\epsilon \sim 10^{-14}$ for $\Lambda \sim M_{\mathrm{Pl}}$, $\langle\Sigma\rangle \sim M_{\mathrm{GUT}}$, and $\left\langle\Sigma^{\prime}\right\rangle \sim 10^{8} \mathrm{GeV}$.

\section{SUMMARY}

Because simple dark matter candidates such as a fourth generation heavy neutrino with mass of order a few $\mathrm{GeV}$, or the lightest supersymmetric particle such as a neutralino with mass of order a few hundred $\mathrm{GeV}$, have been excluded (in the case of the former), and severely constrained (in the case of the latter), a plethora of dark matter candidates have arisen with varying degrees of simplicity. Among these, there are many theories with a presumed stable dark matter candidate which has no SM gauge interactions, and instead carries a charge under some hidden sector gauge group which is often assumed to be $U(1)_{X}$. This opens up the possibility that the gauge field associated with the hidden $U(1)_{X}$, can have a kinetic mixing term with the SM photon.

There is, however, a large body of constraints on the mixing parameter $\epsilon$ which lead to upper limits of order $10^{-7}$ for a wide range of dark photon masses between $\mathcal{O}\left(10^{-14}\right) \mathrm{eV}$ and $\mathcal{O}(100) \mathrm{MeV}$, with significantly stronger bounds $\left(\epsilon<10^{-15}\right)$ for dark photon masses around $1 \mathrm{keV}$ as seen in Fig. 2.

If there are fields which are charged under both the SM and the hidden $U(1)_{X}$, then one expects (barring a finetuning) kinetic mixing at the one-loop level, with a value given by the estimate in Eq. (2), which is not much smaller than $10^{-2}$ and in rather severe disagreement with the experimental limits seen in Fig. 2.

In this paper, we have considered both bottom-up and top-down approaches to building a model with sufficiently small kinetic mixing. The bottom-up approach is necessarily complicated by the fact that fields must be charged under only a single $U(1)$, to avoid one-loop mixing. To this end, we have considered a model based on the right-handed neutrino portal which involves both the SM Higgs and a hidden sector Higgs $H_{X}$. When $H_{X}$ acquires a vev, we can construct a two-loop diagram for mixing above and below the weak scale. Since the kinetic mixing in this case is proportional to unknown SM and hidden Yukawa couplings, the mixing parameter can be tuned to very small values.
We have also argued that gravity alone can lead to kinetic mixing. Though this occurs at the six-loop level, it provides us with a lower limit to $\epsilon$ which can be as large as $10^{-13}$ if the hidden sector Yukawa coupling is of order one and the charge conjugation symmetry is broken in the hidden sector.

We have also considered the construction of kinetic mixing in top-down models where all gauge groups are unified into a single GUT. Once again, the prime difficulty is finding matter representations which are not charged under both the SM and hidden $U(1)_{X}$ gauge groups. Indeed, for the commonly studied $S O(10)$ and $E_{6}$ GUT gauge groups, we found no representations which allow us to escape the estimate in Eq. (2). However, in $E_{7}$, which breaks to $S O(10) \times U(1)_{A} \times U(1)_{B}$, the entire SM 16 which originates in a 27 of $E_{7}$ is uncharged under one linear combination of the two $U(1)$ 's. However, the model must be complicated by choosing the Higgs $\mathbf{1 0}$ from a different $E_{7}$ representation, the smallest being the $\mathbf{1 3 3}$. Models in $E_{8}$ GUTs are also possible.

Finally, we also considered models of the form GUT $\times U(1)_{X}$. In this case, we require a higher-dimensional operator to provide the kinetic mixing. If that operator is mediated by Planck-scale physics, we can expect a suppression of order $M_{\mathrm{GUT}} / M_{\mathrm{Pl}}$ over the one-loop estimate. Higher order suppressions are possible if we employ larger representations to break the GUT [such as the $\mathbf{7 5}$ in the case of $S U(5)]$.

Of course nature has already decided if dark matter resides in a hidden sector and communicates with the visible sector through kinetic mixing. We rely on experimental discovery to confirm or exclude this class of theories. We have seen, however, that the construction of such theories, whether within the context of a GUT or not, is highly nontrivial. Furthermore, kinetic mixing through gravity may already preclude some range of dark photon masses.

\section{ACKNOWLEDGMENTS}

We would like to thank Bohdan Grzadkowski and Jörg Jäckel for helpful discussions. Special thanks are due to Javier Redondo for providing a Mathematica notebook with experimental bounds on kinetic mixing that we used to produce Fig. 2. The work of T. G. and K. A. O. was supported in part by DOE Grant No. DE-SC0011842 at the University of Minnesota. J. K. acknowledges financial support from the Fine Theoretical Physics Institute (FTPI) at the University of Minnesota and the Meltzer Research Fund, and would like to thank the FTPI and the Abdus Salam ICTP (Trieste, Italy), for their hospitality during the work on this project. 
[1] J. E. Gunn, B. W. Lee, I. Lerche, D. N. Schramm, and G. Steigman, Some astrophysical consequences of the existence of a heavy stable neutral lepton, Astrophys. J. 223, 1015 (1978).

[2] ALEPH, DELPHI, L3, OPAL and SLD Collaborations, LEP Electroweak Working Group, SLD Electroweak and Heavy Flavour Groups, Precision electroweak measurements on the $Z$ resonance, Phys. Rep. 427, 257 (2006).

[3] S. P. Ahlen, F. T. Avignone, R. L. Brodzinski, A. K. Drukier, G. Gelmini, and D. N. Spergel, Limits on cold dark matter candidates from an ultralow background Germanium spectrometer, Phys. Lett. B 195, 603 (1987).

[4] D. O. Caldwell, R. M. Eisberg, D. M. Grumm, M. S. Witherell, B. Sadoulet, F. S. Goulding, and A. R. Smith, Laboratory Limits on Galactic Cold Dark Matter, Phys. Rev. Lett. 61, 510 (1988).

[5] M. Beck et al., Searching for dark matter with the enriched detectors of the Heidelberg-Moscow double beta decay experiment, Phys. Lett. B 336, 141 (1994).

[6] H. Goldberg, Constraint on the Photino Mass from Cosmology, Phys. Rev. Lett. 50, 1419 (1983); Erratum 103, 099905 (2009).

[7] J. R. Ellis, J. S. Hagelin, D. V. Nanopoulos, K. A. Olive, and M. Srednicki, Supersymmetric relics from the big bang, Nucl. Phys. B238, 453 (1984).

[8] M. Aaboud et al. (ATLAS Collaboration), Search for supersymmetry in final states with missing transverse momentum and multiple $b$-jets in proton-proton collisions at $\sqrt{s}=13 \mathrm{TeV}$ with the ATLAS detector, J. High Energy Phys. 06 (2018) 107.

[9] M. Aaboud et al. (ATLAS Collaboration), Search for squarks and gluinos in final states with jets and missing transverse momentum using $36 \mathrm{fb}^{-1}$ of $\sqrt{s}=13 \mathrm{TeV}$ pp collision data with the ATLAS detector, Phys. Rev. D 97, 112001 (2018).

[10] A. M. Sirunyan et al. (CMS Collaboration), Search for new phenomena with the $M_{\mathrm{T} 2}$ variable in the all-hadronic final state produced in proton-proton collisions at $\sqrt{s}=13 \mathrm{TeV}$, Eur. Phys. J. C 77, 710 (2017).

[11] A. M. Sirunyan et al. (CMS Collaboration), Search for natural and split supersymmetry in proton-proton collisions at $\sqrt{s}=13 \mathrm{TeV}$ in final states with jets and missing transverse momentum, J. High Energy Phys. 05 (2018) 025.

[12] D. S. Akerib et al. (LUX Collaboration), Results from a Search for Dark Matter in the Complete LUX Exposure, Phys. Rev. Lett. 118, 021303 (2017).

[13] X. Cui et al. (PandaX-II Collaboration), Dark Matter Results from 54-Ton-Day Exposure of PandaX-II Experiment, Phys. Rev. Lett. 119, 181302 (2017).

[14] E. Aprile et al. (XENON Collaboration), Dark Matter Search Results from a One Ton-Year Exposure of XENON1T, Phys. Rev. Lett. 121, 111302 (2018).

[15] J. Preskill, M. B. Wise, and F. Wilczek, Cosmology of the invisible axion, Phys. Lett. 120B, 127 (1983).

[16] L. F. Abbott and P. Sikivie, A cosmological bound on the invisible axion, Phys. Lett. 120B, 133 (1983).

[17] M. Dine and W. Fischler, The not so harmless axion, Phys. Lett. 120B, 137 (1983).

[18] J. Jaeckel and A. Ringwald, The low-energy frontier of particle physics, Annu. Rev. Nucl. Part. Sci. 60, 405 (2010).
[19] J. Jaeckel, A force beyond the Standard Model-Status of the quest for hidden photons, Frascati Phys. Ser. 56, 172 (2012).

[20] J. Alexander et al., Dark Sectors 2016 Workshop: Community Report (2016), http://lss.fnal.gov/archive/2016/conf/ fermilab-conf-16-421.pdf.

[21] M. Battaglieri et al., US cosmic visions: New ideas in dark matter 2017: Community report, in U.S. Cosmic Visions: New Ideas in Dark Matter College Park, MD, USA (2017), http://lss.fnal.gov/archive/2017/conf/fermilab-conf-17-282ae-ppd-t.pdf.

[22] J. Beacham et al., Physics beyond colliders at CERN: Beyond the Standard Model working group report, arXiv:1901.09966.

[23] A. Alves, S. Profumo, and F. S. Queiroz, The dark $Z^{\prime}$ portal: Direct, indirect and collider searches, J. High Energy Phys. 04 (2014) 063.

[24] O. Lebedev and Y. Mambrini, Axial dark matter: The case for an invisible $Z^{\prime}$, Phys. Lett. B 734, 350 (2014).

[25] G. Arcadi, Y. Mambrini, M. H. G. Tytgat, and B. Zaldivar, Invisible $Z^{\prime}$ and dark matter: LHC vs LUX constraints, J. High Energy Phys. 03 (2014) 134.

[26] B. Holdom, Two U(1)'s and $\epsilon$ charge shifts, Phys. Lett. 166B, 196 (1986).

[27] C. Cheung, J. T. Ruderman, L.-T. Wang, and I. Yavin, Kinetic mixing as the origin of light dark scales, Phys. Rev. D 80, 035008 (2009).

[28] V. Popov, On the experimental search for photon mixing, Turk. J. Phys. 23, 943 (1999).

[29] J. Jaeckel, J. Redondo, and A. Ringwald, Signatures of a Hidden Cosmic Microwave Background, Phys. Rev. Lett. 101, 131801 (2008).

[30] A. Mirizzi, J. Redondo, and G. Sigl, Microwave background constraints on mixing of photons with hidden photons, J. Cosmol. Astropart. Phys. 03 (2009) 026.

[31] E. R. Williams, J. E. Faller, and H. A. Hill, New Experimental Test of Coulomb's Law: A Laboratory Upper Limit on the Photon Rest Mass, Phys. Rev. Lett. 26, 721 (1971).

[32] M. Betz, F. Caspers, M. Gasior, M. Thumm, and S. W. Rieger, First results of the CERN resonant weakly interacting sub-eV particle search (CROWS), Phys. Rev. D 88, 075014 (2013).

[33] P. W. Graham, J. Mardon, S. Rajendran, and Y. Zhao, Parametrically enhanced hidden photon search, Phys. Rev. D 90, 075017 (2014).

[34] H. An, M. Pospelov, and J. Pradler, New stellar constraints on dark photons, Phys. Lett. B 725, 190 (2013).

[35] J. Redondo and G. Raffelt, Solar constraints on hidden photons re-visited, J. Cosmol. Astropart. Phys. 08 (2013) 034.

[36] N. Vinyoles, A. Serenelli, F. L. Villante, S. Basu, J. Redondo, and J. Isern, New axion and hidden photon constraints from a solar data global fit, J. Cosmol. Astropart. Phys. 10 (2015) 015.

[37] J. Redondo and M. Postma, Massive hidden photons as lukewarm dark matter, J. Cosmol. Astropart. Phys. 02 (2009) 005.

[38] J. D. Bjorken, R. Essig, P. Schuster, and N. Toro, New fixedtarget experiments to search for dark gauge forces, Phys. Rev. D 80, 075018 (2009).

[39] J. Redondo, Atlas of solar hidden photon emission, J. Cosmol. Astropart. Phys. 07 (2015) 024. 
[40] A. Fradette, M. Pospelov, J. Pradler, and A. Ritz, Cosmological constraints on very dark photons, Phys. Rev. D 90, 035022 (2014).

[41] B. Batell, M. Pospelov, and A. Ritz, Exploring portals to a hidden sector through fixed targets, Phys. Rev. D 80, 095024 (2009).

[42] E. Izaguirre, G. Krnjaic, P. Schuster, and N. Toro, New electron beam-dump experiments to search for $\mathrm{MeV}$ to fewGeV dark matter, Phys. Rev. D 88, 114015 (2013).

[43] M. Ahlers, J. Jaeckel, J. Redondo, and A. Ringwald, Probing hidden sector photons through the Higgs window, Phys. Rev. D 78, 075005 (2008).

[44] C. P. Burgess, J. P. Conlon, L.-Y. Hung, C. H. Kom, A. Maharana, and F. Quevedo, Continuous global symmetries and hyperweak interactions in string compactifications, J. High Energy Phys. 07 (2008) 073.

[45] M. Cicoli, M. Goodsell, J. Jaeckel, and A. Ringwald, Testing string vacua in the lab: From a hidden CMB to dark forces in flux compactifications, J. High Energy Phys. 07 (2011) 114.

[46] K. R. Dienes, C. F. Kolda, and J. March-Russell, Kinetic mixing and the supersymmetric gauge hierarchy, Nucl. Phys. B492, 104 (1997).

[47] P. G. Cámara, L. E. Ibáñez, and F. Marchesano, RR photons, J. High Energy Phys. 09 (2011) 110.

[48] M. Bianchi, G. Inverso, J. F. Morales, and D. R. Pacifici, Unoriented quivers with flavour, J. High Energy Phys. 01 (2014) 128.

[49] M. Garny, A. Palessandro, M. Sandora, and M. S. Sloth, Charged planckian interacting dark matter, J. Cosmol. Astropart. Phys. 01 (2019) 021.

[50] A. Banerjee, G. Bhattacharyya, D. Chowdhury, and Y. Mambrini, Dark matter seeping through dynamic gauge kinetic mixing, arXiv:1905.11407.

[51] Z. Chacko, H.-S. Goh, and R. Harnik, Natural Electroweak Breaking from a Mirror Symmetry, Phys. Rev. Lett. 96, 231802 (2006).

[52] S. Koren and R. McGehee, Freeze-twin dark matter, arXiv:1908.03559.

[53] M. Pospelov, Neutrino physics with dark matter experiments and the signature of new Baryonic neutral currents, Phys. Rev. D 84, 085008 (2011).
[54] B. Dasgupta and J. Kopp, Cosmologically Safe eV-Scale Sterile Neutrinos and Improved Dark Matter Structure, Phys. Rev. Lett. 112, 031803 (2014).

[55] T. Bringmann, J. Hasenkamp, and J. Kersten, Tight bonds between sterile neutrinos and dark matter, J. Cosmol. Astropart. Phys. 07 (2014) 042.

[56] D. Dunsky, L. J. Hall, and K. Harigaya, Higgs parity, strong $C P$, and dark matter, J. High Energy Phys. 07 (2019) 016.

[57] H. An, M. Pospelov, J. Pradler, and A. Ritz, Direct detection constraints on dark photon dark matter, Phys. Lett. B 747, 331 (2015).

[58] G. Dvali, Black holes and large $\mathrm{N}$ species solution to the hierarchy problem, Fortschr. Phys. 58, 528 (2010).

[59] N. Arkani-Hamed, T. Cohen, R. T. D’Agnolo, A. Hook, H. D. Kim, and D. Pinner, Solving the Hierarchy Problem at Reheating with a Large Number of Degrees of Freedom, Phys. Rev. Lett. 117, 251801 (2016).

[60] G. F. Giudice and M. McCullough, A clockwork theory, J. High Energy Phys. 02 (2017) 036.

[61] B. Batell and T. Gherghetta, Localized U(1) gauge fields, millicharged particles, and holography, Phys. Rev. D 73, 045016 (2006).

[62] R. Slansky, Group theory for unified model building, Phys. Rep. 79, 1 (1981).

[63] R. Feger and T. W. Kephart, LieART-A mathematica application for Lie algebras and representation theory, Comput. Phys. Commun. 192, 166 (2015).

[64] J. Sayre, S. Wiesenfeldt, and S. Willenbrock, Minimal trinification, Phys. Rev. D 73, 035013 (2006).

[65] N. Arkani-Hamed and N. Weiner, LHC signals for a SuperUnified theory of dark matter, J. High Energy Phys. 12 (2008) 104.

[66] A. Masiero, D. V. Nanopoulos, K. Tamvakis, and T. Yanagida, Naturally massless Higgs doublets in supersymmetric SU(5), Phys. Lett. 115B, 380 (1982).

[67] B. S. Acharya, S. A. R. Ellis, G. L. Kane, B. D. Nelson, and M. Perry, Categorisation and detection of dark matter candidates from string/M-theory hidden sectors, J. High Energy Phys. 09 (2018) 130.

[68] H. Goldberg and L. J. Hall, A new candidate for dark matter, Phys. Lett. B 174, 151 (1986). 